\title{
Errors on the Trail Making Test Are Associated with Right Hemispheric Frontal Lobe Damage in Stroke Patients
}

\author{
Bruno Kopp, ${ }^{1,2}$ Nina Rösser, ${ }^{1}$ Sandra Tabeling, ${ }^{1,3}$ Hans Jörg Stürenburg, ${ }^{3}$ Bianca de Haan, \\ Hans-Otto Karnath, ${ }^{4,5}$ and Karl Wessel ${ }^{1}$ \\ ${ }^{1}$ Cognitive Neurology, Technische Universität Braunschweig and Department of Neurology, Braunschweig Hospital, \\ Salzdahlumer Street 90, 38126 Braunschweig, Germany \\ ${ }^{2}$ Department of Neurology, Hannover Medical School, Carl-Neuberg Street 1, 30625 Hannover, Germany \\ ${ }^{3}$ Klinik Niedersachsen, Haupt Street 59, 31542 Bad Nenndorf, Germany \\ ${ }^{4}$ Division of Neuropsychology, Center of Neurology, Hertie Institute for Clinical Brain Research, University of Tübingen, \\ Hoppe-Seyler Street 3, 72076 Tübingen, Germany \\ ${ }^{5}$ Department of Psychology, University of South Carolina, 1512 Pendleton Street, Columbia, SC 29208, USA
}

Correspondence should be addressed to Karl Wessel; k.wessel@klinikum-braunschweig.de

Received 30 January 2015; Revised 20 March 2015; Accepted 15 April 2015

Academic Editor: Marjan Jahanshahi

Copyright (C) 2015 Bruno Kopp et al. This is an open access article distributed under the Creative Commons Attribution License, which permits unrestricted use, distribution, and reproduction in any medium, provided the original work is properly cited.

\begin{abstract}
Measures of performance on the Trail Making Test (TMT) are among the most popular neuropsychological assessment techniques. Completion time on TMT- $A$ is considered to provide a measure of processing speed, whereas completion time on TMT- $B$ is considered to constitute a behavioral measure of the ability to shift between cognitive sets (cognitive flexibility), commonly attributed to the frontal lobes. However, empirical evidence linking performance on the TMT-B to localized frontal lesions is mostly lacking. Here, we examined the association of frontal lesions following stroke with TMT-B performance measures (i.e., completion time and completion accuracy measures) using voxel-based lesion-behavior mapping, with a focus on right hemispheric frontal lobe lesions. Our results suggest that the number of errors, but not completion time on the TMT- $B$, is associated with right hemispheric frontal lesions. This finding contradicts common clinical practice-the use of completion time on the TMT-B to measure cognitive flexibility, and it underscores the need for additional research on the association between cognitive flexibility and the frontal lobes. Further work in a larger sample, including left frontal lobe damage and with more power to detect effects of right posterior brain injury, is necessary to determine whether our observation is specific for right frontal lesions.
\end{abstract}

\section{Introduction}

Trail making tasks are popular neuropsychological tests $[1,2]$, because of their ease of administration and the presumed utility as sensitive measures of brain dysfunction [3]. The most widespread trail making task is Trail Making Test, Parts $A$ and $B$ (TMT; [4]). On TMT-A, one connects 25 encircled numbers randomly arranged on a page in ascending order by drawing a pencil line (i.e., 1-2-3 $\cdots 25$ ). It is the same on $T M T-B$, except that 25 encircled numbers and letters need to be connected in alternating order (i.e., 1-A-2 $\cdots 12$-L-13).

Trail making tasks were originally based on a test measuring divided attention, Partington's Pathways [5]. Halstead [6] recognized its potential for his studies of the biological basis for intelligence. Many other trail making tasks are available meanwhile (e.g., [7]; see [3], for review). Perceptual/motor speed, speed of cognitive processing, (divided) attention, visual search, working memory, executive control, cognitive flexibility, and general intelligence contribute to TMT performance. However, there is no consensus about their exact nature and relative contributions (see [8] for review). There exists a substantial correlation between TMT- $A$ and TMT-B completion times [9], suggesting that they measure "... similar although somewhat different functions" [3, page 668]. Executive abilities (see [10], for discussion) play an important role in TMT-B performance. According to Kortte et al. [11], TMT-B performance correlates more closely with cognitive flexibility measures (i.e., perseveration errors on the 
Wisconsin Card Sorting Test) (WCST [12]; see [13], for review) than with working memory measures (i.e., failure to maintain set score on WCST). The consistent finding of substantial correlations between TMT-B and WCST perseverative indices suggests cognitive flexibility being among the key executive abilities underlying performance on the TMT-B (e.g., [11]).

TMT completion time measures are sensitive to the presence of various neurological and psychiatric disorders [14], but their diagnostic utility in differential diagnosis has repeatedly been questioned [15-17]. The generally accepted linkage between cognitive flexibility and frontal lobe functioning (e.g., [18]) suggests that the TMT-B could be used to evaluate frontal lobe (dys)function. Ricker et al. [19] found TMT-B completion time being related to frontal lobe dysfunction. However, this finding must be treated cautiously given negative results in studies comparing patients with frontal and posterior brain damage on TMT-B completion time $[20,21]$. A meta-analysis by Demakis [22] found significant group differences only for completion time on TMT-A, but the effect size was small, indicating little separation between frontal and posterior groups and relatively poor TMT-A sensitivity and specificity. Bonilha et al. [23] investigated the relationship between prefrontal cortical atrophy and neuropsychological performance in schizophrenic patients and found that decreased Brodmann's area (BA9) grey matter volume correlated with poorer task performance on WCST errors and TMT-B completion time.

Taken together, the association between TMT-B completion time measures and frontal lobe dysfunction seems to be relatively weak or even absent. Against this background, it is often thought that prolongation of TMT-B completion time in the presence of normal TMT-A completion time does suggest frontal dysfunction. Specifically, subtracting TMT$A$ from TMT-B completion time is a common method for partialling out effects of general processing speed difficulties that patients might have [1]. Although this subtraction method is widely used in clinical practice, we deliberately decided to refrain from examining whether TMT-B minus TMT-A completion time data correlate with relevant brain regions. Our reluctance can be traced back to the fact that the difference between two substantially correlated measures, as in the case of TMT-A and TMT-B completion times, possesses unacceptable low levels of reliability, thereby precluding its potential use in clinical practice (see [9], for the rationale behind this recommendation).

Thus, there remains a need for additional research on potential associations between cognitive flexibility, as assessed by the TMT, and frontal lobes. TMT-B completion accuracy represents a promising candidate measure in that regard since a former study suggested a relationship between TMT-B completion accuracy measures and frontal brain dysfunctions [24]. Analysis of errors on TMT-B in Stuss et al.s [24] study indicated that all patients who made two or more than two errors had frontal lesions (but see [17], for a failure to replicate Stuss et al.'s [24] finding). Further, dividing the frontal damaged patients into subgroups on the basis of the number of errors yielded specificity of brain-behavior relations within the frontal lobes: patients with damage in dorsolateral frontal areas were most impaired, while those with damage to the medial frontal lobes were not significantly affected on TMT-B completion accuracy.

Klusman et al. [25] distinguished between TMT-B shifting (e.g., connecting $1-2 \cdots$ or $\mathrm{A}-\mathrm{B} \cdots$ ) and sequencing errors (e.g., connecting $1-\mathrm{A}-3 \cdots$ or A-2-C...). Stuss et al. [24] found notable slowing of TMT-B in patients with frontal lobe damage, but they concluded error analysis providing a more useful method to differentiate between frontal and posterior brain damage because all patients committing two or more TMT-B errors had frontal lesions. Notably, many patients with lesions in the dorsolateral prefrontal cortex (DLPFC) committed two or more errors (irrespective of error type), whereas ventrolateral prefrontal and orbitofrontal lesions did not affect TMT-B accuracy variables comparably. Stuss and Levine [18, page 415] concluded the following: "TMT-B errors (but not time), therefore, are a valid measure of DLPFC dysfunction."

In the present study, we investigate the association between TMT completion time and accuracy measures with frontal lobe damage in stroke patients using voxel-based lesion-behavior mapping [26-28]. In contrast to traditional overlap designs [26] in which the overlapping of lesion boundaries in individual patients from different groups limits study validity (cf. [26]), voxel-based lesion-behavior analysis yields a statistical approach to uncover brain-behavior relationships without any prior patient categorization. Moreover, previous research on behavioral effects of frontal brain damage often compared patient groups with heterogeneous etiological lesions (see [29]). These shortcomings notwithstanding and based on Stuss et al. [24], we hypothesize that TMT-B completion accuracy measures, but not TMT-B completion time measures, are sensitive to DLPFC damage.

\section{Method}

2.1. Subjects. Thirty acute, first-ever, and right-hemispheredamaged stroke patients with damage involving the frontal lobe in most patients participated in the study (see Table 1). These neuropsychological results show that our sample consisted of stroke patients without generalized cognitive deficits as revealed by the Mini-Mental-State-Examination (MMSE, [32]) and without notable verbal disturbances as revealed by the Wortschatz-Test (Vocabulary Test) (WST, [35]) and by the Regensburger Wortflüssigkeits-Test (Regensburger Word Fluency Test) (RWT, [34]). Further, the Modified Card Sorting Test (MCST, [33]) (a variant of the WCST) data suggest that cognitive flexibility and working memory were not severely disturbed in our patients.

The logic in restricting to right hemispheric strokes was to exclude patients with paresis of the dominant right hand and/or apraxia, possibly distorting task performance. Further, left-hemisphere strokes might have hampered the understanding of task instructions, due to potential presence of sensory aphasia. ${ }^{1}$ Patients with traumatic brain injury, brain tumours, subcortical arteriosclerotic encephalopathy, neurodegenerative disease, or gross neurological defects (pronounced pain reported by the patient, left homonymous hemianopia revealed by clinical examination, and hemispatial visual neglect) were excluded to ensure symptoms did 
TABLE 1: Demographic and neuropsychological patient characteristics.

\begin{tabular}{lccc}
\hline & $N$ & $\mathrm{M}$ & $\mathrm{SD}$ \\
\hline Age & 30 & 60.17 & 9.70 \\
Sex & 30 & $17(\mathrm{~m}) / 13(\mathrm{f})$ & $/$ \\
Years of education & 30 & 12.17 & 2.16 \\
Handedness & 30 & 0.93 & 0.27 \\
CES-D $(z)$ & 26 & 0.10 & 0.86 \\
MMSE $(\mathrm{rs})$ & 30 & 27.50 & 2.18 \\
WST $(z)$ & 28 & -0.36 & 0.83 \\
RWT-subtest s-words $(z)$ & 30 & -0.38 & 0.65 \\
RWT-subtest animals $(z)$ & 30 & -0.29 & 0.51 \\
MCST-N categories $(\mathrm{rs})$ & 26 & 5.42 & 1.21 \\
MCST-N perseveration errors $(\mathrm{rs})$ & 26 & 1.81 & 2.33 \\
\hline
\end{tabular}

Center for Epidemiologic Studies Depression Scale [30]; handedness: handedness ratio on the Edinburgh Handedness Questionnaire [31]; $-1=$ strongly left-handed, 0 = ambidextrous, and 1 = strongly right-handed; Mini-MentalState-Examination [32]; Modified Card Sorting Test [33]; Regensburger Wortflüssigkeits-Test (Regensburger Word Fluency Test) [34]; WortschatzTest (Vocabulary Test) [35].

Note. Sex: $\mathrm{m}=$ male and $\mathrm{f}=$ female; years of education: school and vocational education; $N=$ number of subjects; $\mathrm{M}=$ Mean; $\mathrm{SD}=$ standard deviation; $z=$ standardized $z$-score; rs $=$ raw score.

not interfere with task performance. ${ }^{1}$ Spatial neglect was diagnosed when a patient oriented toward the ipsilesional side when addressed from the front or left and/or ignored contralesionally located people or objects. Patients without prior psychiatric disease, alcohol, or drug abuse were recruited.

In many studies, patients with lesions from many different etiologies are assessed (e.g., patients with traumatic brain injury and brain tumors). Here, we restricted ourselves to acute stroke patients (most often due to infarcts of the middle cerebral artery, a minority with anterior or posterior cerebral artery infarcts or with hemorrhagic stroke) in order to assure that the full neurological damage did not extend considerably beyond the borders of the visible lesion. As a consequence, the cognitive deficits displayed by the majority of our patients are most likely (solely) related to the visible lesion.

All patients gave their informed written consent to participate in the study, in accordance with the ethical standards of the Declaration of Helsinki (1964). Appropriate ethical approval for the study was obtained from the Ethics Committee at Technische Universität Braunschweig (Faculty for Life Sciences; ref. 37-2010).

\subsection{Materials}

2.2.1. Test Description. Each patient performed the TMT$A$ and TMT-B as described above. Before each test trial, a practice trial of six items was administered to ensure task understanding. Participants were instructed to perform the test as fast and as accurately as possible. During performance, each error was immediately corrected by the examiner instructing the participant to go back with his pencil to the last correct item. All participants continued until they completed the task. Raw completion times and number of errors were the dependent measurements.
One error type on TMT-A (i.e., one type of sequencing error) and four error types on TMT-B (i.e., two types of sequencing and shifting errors, resp.) were classified according to Klusman et al. [25]. In making a shifting error, participants failed to alternate correctly either from a number to a letter (Type A error; e.g., 1-A-2- $\underline{3}$; incorrect choices are underlined) or from a letter to a number (Type B error; e.g., 1-A-B). A sequencing error occurred when participants incorrectly sequenced either numbers (Type $C$ error; e.g., 1- $\underline{3}$ on TMT-A or $1-\mathrm{A}-\underline{3}$ on TMT-B) or letters (Type D error; e.g., 1-A-2-C on TMT-B).

2.3. Lesion Analysis. Magnetic resonance imaging (MRI) was performed in 27 stroke patients and computed tomography (spiral CT) scanning in three stroke patients. The initial scanning was repeated until the infarcted area became clearly demarcated. The mean time interval between lesion onset and MRI scan amounted to 3.9 days ( $\mathrm{SD}=3.2$ ), between lesion onset and CT scanning to 2.6 days $(\mathrm{SD}=3.7)$. MRI scans were obtained on a $1.5 \mathrm{~T}$ echo planar imaging (EPI) capable system (Philips Intera, Philips Medical Systems, Best, Netherlands). The MRI protocol used diffusion-weighted imaging (DWI, $N=11$ ) and $\mathrm{T}_{2}$-weighted fluid-attenuated inversion-recovery imaging (FLAIR, $N=16$ ). DWI was performed with a single-shot EPI spin echo sequence (25 axial slices; repetition times (TR), either 3690, 4000, 4452, 5060,5300 , or $6360 \mathrm{~ms}$; echo times (TE), either 90, 95, or $120 \mathrm{~ms}$; field of view (FOV), $230 \times 230 \mathrm{~mm}^{2}$; matrix $64 \times 64$ pixels; slice thickness, $5 \mathrm{~mm}$; gap, $5.5 \mathrm{~mm}$ ). FLAIR sequences were acquired with 25 axial slices (thickness, $5 \mathrm{~mm}$ ) with an interslice gap of $5.5 \mathrm{~mm}$, FOV of $220 \times 220 \mathrm{~mm}^{2}$, TR of either $4000,5397,5500$, or $6000 \mathrm{~ms}$, and TE of either 89, 91, 100, or $120 \mathrm{~ms}$. CTs were obtained on a spiral scanning system (Somatom Sensation 16, Siemens Healthcare, Erlangen, Germany) with a slice thickness of $3 \mathrm{~mm}$ infratentorial and $6 \mathrm{~mm}$ supratentorial (and an in-plane resolution of $0.5 \times 0.5 \mathrm{~mm}$ ).

Lesion location was evaluated using MRIcroN ([27]; http://www.mricro.com/). In MRI scans, lesion boundaries were delineated directly on the individual scans. MRI scan and lesion shape were then mapped into stereotaxic space using the normalization algorithm in SPM5 (http://www.fil.ion.ucl.ac.uk/spm/). Cost-function masking was employed for determination of transformation parameters [36].

In spiral CT scans, lesions were drawn directly by an experienced neurologist (H.-O. K., blinded for test performance) on the slices of a normalized $\mathrm{T}_{1}$-weighted template MRI scan from the Montreal Neurological Institute (MNI) with a $1 \times$ $1 \mathrm{~mm}$ in-plane resolution, distributed with MRIcroN. Lesions were mapped onto the slices that correspond to MNI $Z$ coordinates $(-16,-8,0,8,16,24,32$, and $40 \mathrm{~mm})$ by using the identical or closest matching axial slices of each individual patient.

To evaluate the relationship between lesion location and performance on TMT- $A$ and TMT- $B$, a voxel-based lesionbehavior analysis was performed using the Brunner-Munzel (BM) test [37] for continuous variables implemented in MRIcroN ([27]; http://www.mricro.com/). Only voxels $(N=$ 146.224) damaged in at least three patients were included in 
TABLE 2: Neuropsychological results (number of subjects) and Brunner-Munzel test statistics (max. $z, z_{\text {crit }}$ ) over various TMT error scores.

\begin{tabular}{|c|c|c|c|c|c|}
\hline$T M T$ error scores & no errors & 1 error & $\geq 2$ erorrs & $\max . z$ & $z_{\text {crit }}$ \\
\hline$T M T-A$, sequencing errors (type C) & 26 & 2 & 2 & 2.425 & 3.452 \\
\hline$T M T-B$, total errors & 9 & 8 & $13^{+}$ & $3.972^{*}$ & 3.501 \\
\hline$T M T-B$, shifting errors (type A) & 18 & 9 & 2 & 3.084 & 3.381 \\
\hline$T M T-B$, shifting errors (type B) & 20 & 6 & 4 & $4.163^{*}$ & 3.285 \\
\hline TMT- $B$, shifting errors (type $\mathrm{A}+\mathrm{B}$ ) & 16 & 7 & 7 & $3.233^{*}$ & 2.996 \\
\hline$T M T-B$, sequencing errors (type C) & 28 & 2 & 0 & 3.154 & 3.154 \\
\hline$T M T-B$, sequencing errors (type D) & 22 & 6 & 2 & $4.250^{*}$ & 3.403 \\
\hline$T M T-B$, sequencing errors (type $\mathrm{C}+\mathrm{D}$ ) & 20 & 8 & 2 & $3.724^{*}$ & 3.285 \\
\hline
\end{tabular}

Note: ${ }^{*} p<.05 .{ }^{+}$All patients who made two or more than two errors of either type on the TMT-B (i.e., TMT-B total errors) had frontal lesions in Stuss et al.s [24] study.

TABLE 3: Neuropsychological results and Brunner-Munzel test statistics (max. $z, z_{\text {crit }}$ ) over various TMT time scores.

\begin{tabular}{|c|c|c|c|c|c|c|}
\hline TMT time scores & M & $\mathrm{SD}$ & Mdn & IQR & $\max . z$ & $z_{\text {crit }}$ \\
\hline TMT-A, completion time (sec) & 82.95 & 122.15 & 48.10 & 61.02 & 2.549 & 3.121 \\
\hline TMT-B, completion time (sec) & 184.51 & 153.01 & 134.13 & 114.36 & 3.320 & 3.320 \\
\hline
\end{tabular}

Note: $\mathrm{IQR}=$ interquartile range $\left(\mathrm{Q}_{75}-\mathrm{Q}_{25}\right)$.

the analysis. We controlled for multiple comparisons applying permutation-based thresholding [38] using 4000 iterations. All results presented survived a $5 \%$ permutation-based false positive probability threshold.

\section{Results}

3.1. Neuropsychological Results. Tables 2 and 3 summarize patients' TMT performance. Average completion times showed the typical prolongation of TMT-B compared to $T M T-A$. Sequencing errors on TMT-A occurred relatively infrequently ( $6.7 \%$ of patients committed $\geq 2$ errors). Total errors on TMT-B were much more common ( $43 \%$ of patients committed $\geq 2$ errors). Type B ( $13.3 \%$ of patients committed $\geq 2$ errors) outnumbered Type A (6.7\% of patients committed $\geq 2$ errors) shifting errors, indicating that the failure to shift from a letter to a number was more prevalent than the failure to shift from numbers to letters. Whereas incorrectly sequencing numbers in ascending order hardly ever occurred on TMT-B ( $0 \%$ of patients committed $\geq 2$ errors), the failure to connect letters in correct alphabetical order happened slightly more often ( $6.7 \%$ of patients committed $\geq 2$ errors). Note that these data are merely descriptive and that they are not backed up by statistical analyses.

3.2. Lesion Analysis. Figure 1 shows an overlay lesion plot of all thirty patients in eight axial slices of a standard brain in MNI space. The maximum lesion overlap occurred in the right prefrontal cortex (PFC) where up to eleven patients showed overlapping lesions in single voxels.

Figure 2 demonstrates a lesion subtraction analysis for TMT- $B$ total errors. Figure 2(a) shows the overlay lesion plot of patients committing two or more errors on the TMT- $B$, and the overlay lesion plot of patients committing less than two errors is presented in Figure 2(b). The results of subtracting the group with less than two errors from the group with two or more errors are shown in Figure 2(c). The right frontal lobe was more frequently damaged in patients committing two or more errors on the TMT-B.

A voxel-based lesion-behavior analysis revealed a significant association between voxel damage in the right hemispheric DLPFC and TMT-B total errors (cf. Table 2). The significant voxels' locations are shown in Figure 3(a). The analysis revealed three regions: First, an area around MNI coordinates $X=38, Y=2$, and $Z=24$ in the frontal subgyral white matter underneath cortical area BA6. Second, an area around MNI coordinates $X=34, Y=5$, and $Z=32$ in the frontal subgyral white matter underneath cortical area BA9. Third, an area around MNI coordinates $X=37, Y=17$, and $Z=32$ within the right middle frontal gyrus (cortical area BA9).

Figure 3(b) depicts the voxels' location for which a voxelbased lesion-behavior analysis revealed a significant association between voxel damage and number of TMT-B shifting errors (cf. Table 2). The analysis revealed three regions: First, an area around MNI coordinates $X=37, Y=5$, and $Z=24$ in the frontal subgyral white matter underneath cortical area BA6. Second, an area around MNI coordinates $X=35, Y=6$, and $Z=32$ within the right inferior frontal gyrus underneath cortical area BA9. Third, an area around MNI coordinates $X=37, Y=17$, and $Z=32$ within the right middle frontal gyrus (cortical area BA9).

Figure 3(c) depicts the voxels' location for which the voxel-based lesion-behavior analysis revealed a significant association between voxel damage and number of TMT-B sequencing errors (cf. Table 2). The analysis revealed three regions: First, an area around MNI coordinates $X=38$, $Y=3$, and $Z=24$ in the frontal subgyral white matter underneath cortical area BA6. Second, an area around MNI coordinates $X=34, Y=5$, and $Z=32$ in the frontal subgyral white matter underneath cortical area BA9. Third, an area around MNI coordinates $X=37, Y=17$, and $Z=32$ within the right middle frontal gyrus (cortical area BA9). 


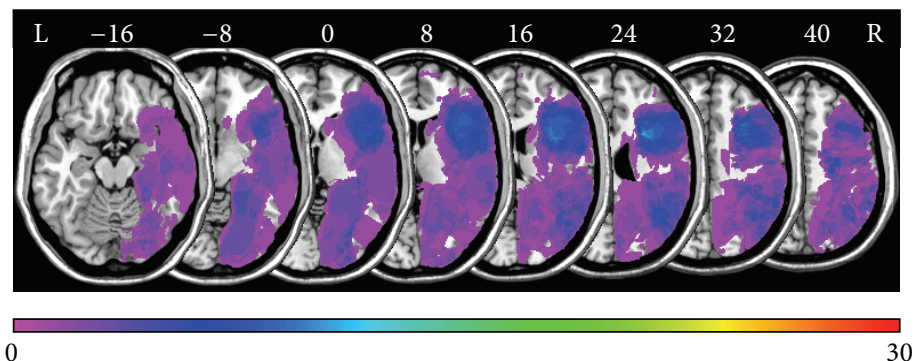

Figure 1: Overlay lesion plots of all thirty patients in MNI space. Eight axial slices. The number of overlapping lesions is illustrated by color, from violet $(N=1)$ to red $(N=30)$. Maximum overlap occurred in the right frontal lobe. The area colored light blue indicates overlapping lesions in eleven patients ( $37 \%$ lesion overlap). Numbers indicate MINI coordinates.

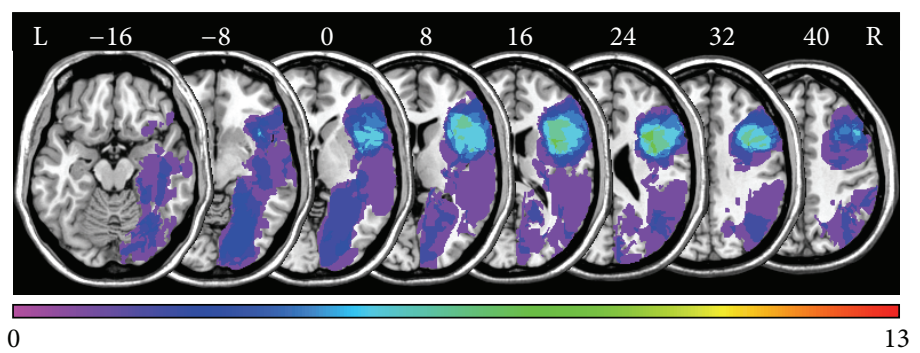

(a)

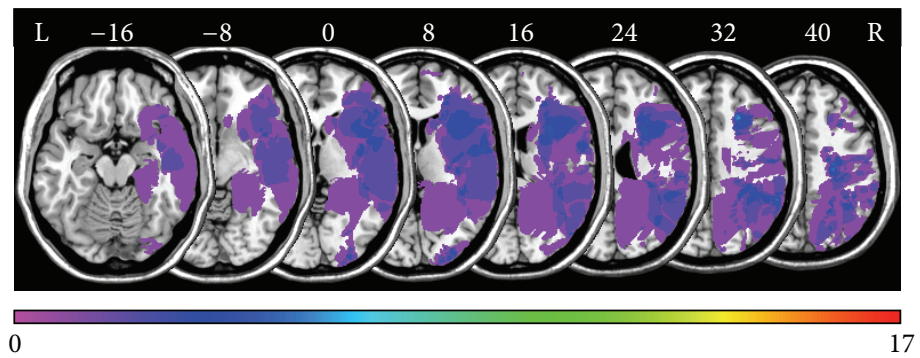

(b)

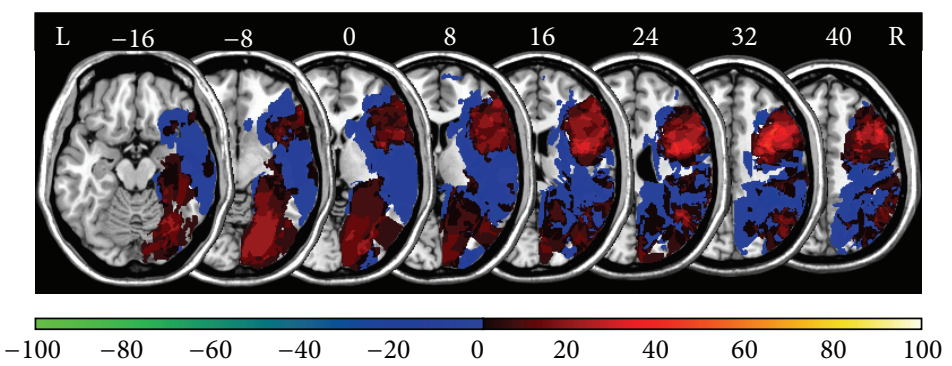

(c)

FIGURE 2: Anatomical results obtained from the lesion subtraction analysis on the number of TMT-B total errors. (a) Overlay lesion plots for those patients who committed two or more total errors $(M d n=1)$ on the TMT-B $(N=13)$. The number of overlapping lesions is illustrated by color, from violet $(N=1)$ to red $(N=13)$. (b) Overlay lesion plots for those patients who committed less than two total errors on the TMT-B $(N=17)$. The number of overlapping lesions is illustrated by color, from violet $(N=1)$ to red $(N=17)$. (c) Overlay plots of the subtracted superimposed lesions of the patients with two or more total errors on the TMT-B minus the patients with less than two total errors on the TMT-B. Colors code increasing frequencies from dark-red (difference from $1 \%$ to $20 \%$ ) to white-yellow (difference from $81 \%$ to $100 \%)$, indicating regions damaged more frequently in patients who committed relatively many total errors on the TMT-B. The colors from dark-blue (difference from -1 to $-20 \%$ ) to light-green (difference from -81 to $-100 \%$ ) indicate regions damaged more frequently in patients who committed relatively few total errors on the TMT-B. 


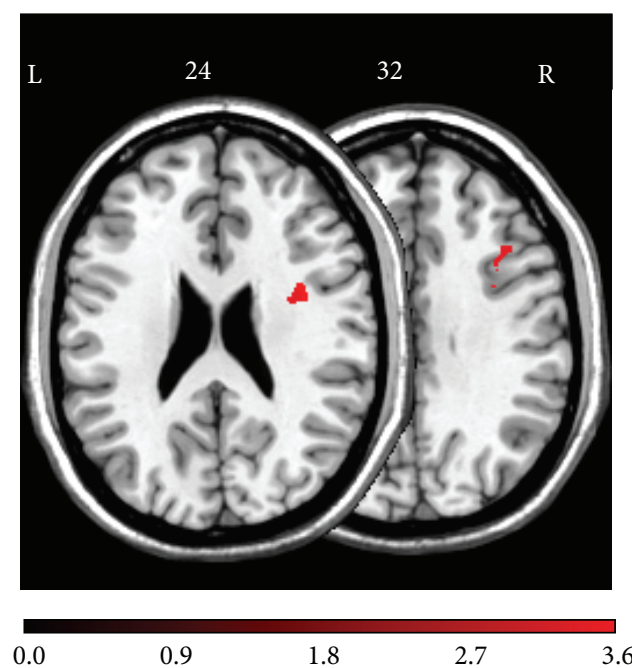

(a)

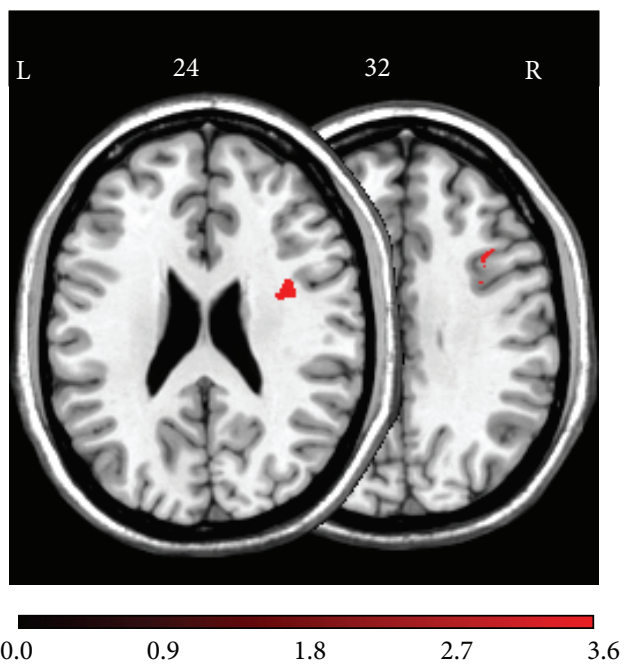

(b)

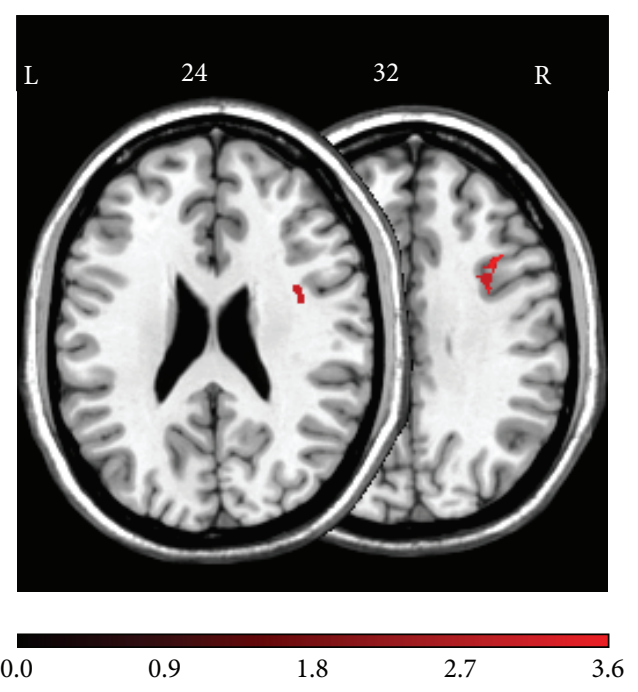

(c)

Figure 3: Anatomical results obtained from the voxel-based lesion-behavior mapping (a) on the number of TMT-B total errors, (b) on the number of TMT-B shifting errors (error type $\mathrm{A}+$ type $\mathrm{B}$ ), and (c) on the number of TMT-B sequencing errors (error type $\mathrm{C}+$ type $\mathrm{D}$ ). The location of voxels for which the voxel-based lesion-behavior mapping indicated that the observed $B z$ surpassed $z_{\text {crit }}$ is shown. See text for details. Numbers indicate MNI coordinates.

No significant association between voxel damage and the total amount of TMT-A errors was found (cf. Table 2).

Voxel-based lesion-behavior analyses revealed no significant association between voxel damage and TMT-A or TMT$B$ completion time (cf. Table 3 ).

\section{Discussion}

Our results are congruent with Stuss et al's [24], according to which TMT-B errors, but not speed measures, are sensitive to DLPFC damage. There are, however, notable differences between these two studies. First, Stuss et al. [24] relied on group analysis treating patients with damage in different brain parts as separate groups. In contrast, voxelbased lesion-behavior mapping analyses whether lesions in individual brain voxels are reliable predictors of behavioral impairments, without any a priori assumptions [26-28]. This improves the anatomical precision for the analysis of lesion location. Second, the patient groups in Stuss et al.s [24] study were quite heterogeneous with regard to lesion etiology and chronicity, two factors which possibly interact in unknown ways with the number of TMT-B errors. In our study, the patient sample was relatively homogeneous, since solely acute stroke patients participated. Further empirical efforts are required to clarify whether the current findings generalize to subacute and chronic stroke patients with prefrontal lesions and to frontal lobe damage of other etiologies. Third, only patients with right hemispheric lesions were included in the current study, whereas patients with lesion in the left or right hemisphere as well as with bilateral lesions participated in Stuss et al.s [24] study. As the number of TMT-B errors was not affected by lesion laterality in this earlier study, 
the authors concluded unilateral prefrontal lesions of either cerebral hemisphere being associated with impaired TMT-B accuracy.

However, this conclusion should be treated cautiously given the available task switching studies. Generally, in task switching experiments, response times (RTs) are slower and response accuracy is often lower for trials requiring task switching compared to task repetition (see [39], for review). Neuropsychological studies of task switching are scarce and their results are contradictory (see [40], for review), possibly due to usually small neurological patient samples. Aron et al. [41] applied a predictable task switching paradigm without explicit task cues (i.e., an endogenous paradigm). Patients with left in contrast to right lateral prefrontal lesions showed significantly larger RT switch costs, whereas the right compared to the left lateral prefrontal group showed dramatically elevated shifting errors on switch trials. Shallice et al. [42] used a task switching paradigm with explicit task cues (i.e., an exogenous paradigm). The authors reported a left lateral prefrontal effect, but this time on errors, whereas the major RT effect was a striking slowing on switch and repeat trials for patients with superior medial prefrontal lesions.

The TMT-B should be considered to be an endogenous task switching paradigm. Given our, Stuss et al.s [24], and Aron et al's [41] results, it has been more consistently demonstrated that lateral prefrontal lesions in the right cerebral hemisphere are associated with elevated error rates in endogenous task switching paradigms. However, this lateralization hypothesis (right/left hemispheric lesions are associated with elevated error rates in endogenous/exogenous task switching paradigms) calls for further systematic inquiry.

Our results show that TMT-B errors, but not completion times, are associated with DLPFC lesions. Most patients who were included in our study had prefrontal lesions, because we were mainly interested in precisely describing prefrontal areas involved in endogenous set shifting. Our study was not specifically designed to detect reliable brain-behaviorrelationships of other, for example, posterior, brain regions. Thus, we do not claim that the documented association between TMT-B performance accuracy and prefrontal lesions is exclusively related to this particular lesion location; rather, the specificity of the prefrontal brain-behavior-relationship has still to be studied. Nevertheless, the relationship between right frontal damage and errors on the TMT-B is of importance against the background that there are few measures available for assessing functional disability in right frontal patients $[10,43,44]$. For example, while verbal fluency can be considered a test of left frontal function $[45,46]$, nonverbal analogs of verbal fluency, such as design fluency, do not seem to provide comparably sensitive and specific indices of right frontal function [47].

We conclude that acute stroke lesions in the right hemispheric DLPFC (i.e., two regions within the right inferior frontal gyrus (BA9 and BA6)) are associated with enhanced $T M T-B$ error proneness (i.e., increased TMT-B total and sequencing errors). Furthermore, a region within the right middle frontal gyrus (BA9) predicted the occurrence of TMT$B$ shifting errors. These anatomical data suggest caudal areas within BA9 and rostral areas within BA6 being frontal regions associated with shifting and sequencing errors on the TMT$B$. In accordance with Stuss et al. [24], the observation of multiple TMT-B errors constitutes a behavioral corollary of right hemisphere DLPFC dysfunction. This is remarkable if one considers that there are not many neuropsychological measures possessing documented sensitivity to right hemispheric DLPFC dysfunction $[18,44]$.

Most of the regions found to be significant from voxelbased lesion-behavior analyses were white matter regions. The importance of white matter disconnection as a pathogenetic mechanism for specific neurological syndromes has been appreciated since long times (e.g., [48]). Our results indicate that white matter disconnection is important for poststroke behavioral deficits. The disconnection of white matter leads to remote physiological effects in regions of cortex that are structurally intact. The cortical distribution of these secondary cortical abnormalities, including the DLPFC, may partly account for the observed behavioral correlations. It is well-established that the DLPFC is implicated as a key node in a frontoparietal network mediating top-down executive control of attention [49]. Damage to the DLPFC, or of white matter beneath the DLPFC, might contribute to the observed behavioral correlations in a variety of ways, as discussed below.

The empirical finding that associates right hemispheric DLPFC lesions with TMT- $B$ errors needs explanation by a cognitive theory. First, a major component of the TMT-B is shifting between two cognitive sets (numbers and letters). Any failure of cognitive set shifting should lead to a specific accumulation of shifting errors. Second, the TMT- $B$ requires serial organization of behavior, under conditions of divided attention and high working memory load. Impaired serial organization of behavior should lead to an accumulation of sequencing errors. However, we observed both, more shifting and more sequencing errors in patients with right hemispheric DLPFC lesions, suggesting that neither a failure of cognitive flexibility nor a failure of serial organization of behavior accounts for our findings. Alternatively, enhanced $T M T-B$ error proneness might result from a more general tendency towards stimulus-bound behavior [10]. From this perspective, both error types reflect "false alarms" to distractors via impaired concentration, enhanced distractibility, and/or disturbed response inhibition, that is, neuropsychological dysfunctions, which are often asserted to right hemispheric DLPFC lesions [50, 51].

\section{Limitations and Conclusions}

The data presented here need to be interpreted with caution mainly for three reasons: First, the majority of the patients in the current sample had right frontal lesions, and an extended sample should include both patients with right and left frontal lobe damage in order to examine whether or not our observation is specific for right frontal lesions. Second, the extended sample should also include many more patients with right posterior damage in order to examine whether or not our observation is specific for frontal lesions. With regard to this issue it would be interesting to know to what extent 
the increased TMT-B error rate in our patients is explained partly by concurrent posterior cortical lesions causing a visuoperceptual deficit. As noted by one of our reviewers, the area of overlapping occipital damage amongst patients was larger and more diffuse than that in prefrontal areas. Thus, our results were obtained in an analysis that was weighted towards highlighting the more concentrated (prefrontal) area. However, damage to multiple sites across the occipital cortex could lead to the same (or similar) visuoperceptual deficit, yet not be highlighted by the analysis used as the lesion sites are more distributed spatially amongst patients. Third, exclusion of patients with visual field defects or with hemispatial neglect generally reduces the generalizability of our claims.

Taken together, our findings suggest that aspects of TMT performance, namely, the accuracy on TMT-B, are associated with right frontal lobe damage. However, all our patients suffered from damage to the right frontal lobe and we can thus not compare the performance of patients with damage to the right frontal lobe to the performance of patients with damage elsewhere. We can, as a consequence, not draw firm conclusions concerning the specificity of the relationship between damage to the right frontal lobe and the number of errors on TMT-B. Specifically, future work should examine $T M T-B$ accuracy in patients with left frontal lesions and in patients with posterior lesions.

On a pragmatic basis, our data imply that one should be cautious about concluding the location of a lesion based on completion times in trail making tasks. However, TMT ranks fourth among the top ten executive functioning instruments [1], and completion times, but not error scores, received standardization [3]. The subtraction method (TMT$B$ completion time minus TMT-A completion time) is widely used in clinical practice; however, the subtraction method should not be pursued for psychometric reasons [9]. The current data suggest that multiple TMT-B errors might be a more sensitive indicator of DLPFC dysfunction, as originally conjectured by Stuss and Levine [18]. The finding that right hemispheric DLPFC lesions were associated with enhanced shifting and sequencing errors alike suggests that rather nonspecific attentional impairments underlie enhanced error proneness, as discussed above.

\section{Abbreviations}

BA: Brodmann's area

BM: Brunner-Munzel test

CES-D: Center for Epidemiologic Studies Depression Scale

CT: $\quad$ Computed tomography

DLPFC: Dorsolateral prefrontal cortex

DWI: Diffusion-weighted imaging

EPI: Echo planar imaging

FLAIR: Fluid-attenuated inversion-recovery imaging

FOV: $\quad$ Field of view

MCST: Modified Card Sorting Test

MMSE: Mini-Mental-State-Examination

MNI: Montreal Neurological Institute
MRI: Magnetic resonance imaging

PFC: Prefrontal cortex

RT: Response time

RWT: Regensburger Wortflüssigkeits-Test

(Regensburger Word Fluency Test)

TE: Echo times

TR: Repetition time

TMT: Trail Making Test

WCST: Wisconsin Card Sorting Test

WST: Wortschatz-Test (Vocabulary Test).

\section{Conflict of Interests}

The authors declare that there is no conflict of interests regarding the publication of this paper.

\section{Acknowledgments}

This work was supported by the ZNS-Hannelore Kohl Stiftung, Bonn, Germany (Grant no. 2004007 to Bruno Kopp and Karl Wessel), by the Erwin-Röver-Stiftung, Hannover, Germany (Grant no. 20082014 to Karl Wessel), and by the Deutsche Forschungsgemeinschaft (Grant nos. KA1258/151 and HA 58393/3-1 to Hans-Otto Karnath and Bianca de Haan).

\section{Endnotes}

1. A possible statistical solution to the problem would be to use the severity of hemiparesis, apraxia, aphasia, pain, hemianopia, neglect, and other neuropsychological disturbances as covariates. However, covariance analysis presupposes the separation of patients into meaningful groups of individuals, as in neuropsychological group studies, and it further requires a number of restrictive conditions to be met, for example, that the slopes of the regression lines (which relate covariates and dependent variables) fitted to the groups, to be parallel.

\section{References}

[1] E. Strauss, E. Sherman, and O. Spreen, A Compendium of Neuropsychological Tests: Administration, Norms, and Commentary, Oxford University Press, New York, NY, USA, 2006.

[2] M. N. Mitrushina, K. B. Boone, J. Razani, and L. F. D'Elia, Handbook of Normative Data for Neuropsychological Assessment, Oxford University Press, New York, NY, USA, 2nd edition, 2005.

[3] R. M. Reitan and D. Wolfson, "A selective and critical review of neuropsychological deficits and the frontal lobes," Neuropsychology Review, vol. 4, no. 3, pp. 161-198, 1994.

[4] R. M. Reitan, "The relation of the Trail Making Test to organic brain damage," Journal of Consulting Psychology, vol. 19, no. 5, pp. 393-394, 1955.

[5] J. E. Partington and R. G. Leiter, "Partington's pathways test," The Psychological Services Center Bulletin, vol. 1, pp. 9-20, 1949.

[6] W. C. Halstead, Brain and Intelligence: A Quantitative Study of the Frontal Lobes, University of Chicago Press, Chicago, Ill, USA, 1947. 
[7] B. Kopp, N. Rösser, and K. Wessel, "Psychometric characteristics and practice effects of the Brunswick Trail Making Test," Perceptual \& Motor Skills, vol. 107, no. 3, pp. 707-733, 2008.

[8] I. Sánchez-Cubillo, J. A. Periáñez, D. Adrover-Roig et al., "Construct validity of the Trail Making Test: role of taskswitching, working memory, inhibition/interference control, and visuomotor abilities," Journal of the International Neuropsychological Society, vol. 15, no. 3, pp. 438-450, 2009.

[9] B. Kopp, "Neuropsychologists must keep their eyes on the reliability of difference measures," Journal of the International Neuropsychological Society, vol. 17, no. 3, pp. 562-563, 2011.

[10] M. D. Lezak, Neuropsychological Assessment, Oxford University Press, New York, NY, USA, 3rd edition, 1995.

[11] K. B. Kortte, M. D. Horner, and W. K. Windham, "The trail making test, part B: cognitive flexibility or ability to maintain set?" Applied Neuropsychology, vol. 9, no. 2, pp. 106-109, 2002.

[12] R. K. Heaton, G. J. Chelune, J. L. Talley, G. G. Kay, and G. Curtiss, Wisconsin Card Sorting Test [Manual], Psychological Assessment Resources, Lutz, Fla, USA, 1993.

[13] G. J. Demakis, "A meta-analytic review of the sensitivity of the Wisconsin Card Sorting Test to frontal and lateralized frontal brain damage," Neuropsychology, vol. 17, no. 2, pp. 255-264, 2003.

[14] C. R. Bowie and P. D. Harvey, "Administration and interpretation of the Trail Making Test," Nature Protocols, vol. 1, no. 5, pp. 2277-2281, 2006.

[15] G. L. Iverson, R. T. Lange, and M. D. Franzen, "Effects of mild traumatic brain injury cannot be differentiated from substance abuse," Brain Injury, vol. 19, no. 1, pp. 11-18, 2005.

[16] E. Tamez, J. Myerson, L. Morris, D. A. White, C. Baum, and L. T. Connor, "Assessing executive abilities following acute stroke with the trail making test and digit span," Behavioural Neurology, vol. 24, no. 3, pp. 177-185, 2011.

[17] E. Chan, S. E. MacPherson, G. Robinson et al., "Limitations of the trail making test part- $\mathrm{B}$ in assessing frontal executive dysfunction," Journal of the International Neuropsychological Society, vol. 21, no. 2, pp. 169-174, 2015.

[18] D. T. Stuss and B. Levine, "Adult clinical neuropsychology: lessons from studies of the frontal lobes," Annual Review of Psychology, vol. 53, pp. 401-433, 2002.

[19] J. H. Ricker, B. N. Axelrod, and B. D. Houtler, "Clinical validation of the oral Trail Making Test," Neuropsychiatry, Neuropsychology and Behavioral Neurology, vol. 9, no. 1, pp. 5053, 1996.

[20] R. M. Reitan and D. Wolfson, "Category test and Trial Making Test as measures of frontal lobe functions," The Clinical Neuropsychologist, vol. 9, no. 1, pp. 50-56, 1995.

[21] D. T. Stuss, D. F. Benson, E. F. Kaplan, W. S. Weir, and C. Della Malva, "Leucotomized and nonleucotomized schizophrenics: comparison on tests of attention," Biological Psychiatry, vol. 16, no. 11, pp. 1085-1100, 1981.

[22] G. J. Demakis, "Frontal lobe damage and tests of executive processing: a meta-analysis of the Category Test, Stroop Test, and Trail-Making Test," Journal of Clinical and Experimental Neuropsychology, vol. 26, no. 3, pp. 441-450, 2004.

[23] L. Bonilha, C. Molnar, M. D. Horner et al., "Neurocognitive deficits and prefrontal cortical atrophy in patients with schizophrenia," Schizophrenia Research, vol. 101, no. 1-3, pp. 142-151, 2008.

[24] D. T. Stuss, S. M. Bisschop, M. P. Alexander, B. Levine, D. Katz, and D. Izukawa, "The trail making test: a study in focal lesion patients," Psychological Assessment, vol. 13, no. 2, pp. 230-239, 2001.

[25] L. E. Klusman, L. I. Cripe, and C. B. Dodrill, "Analysis of errors on the Trail Making Test," Perceptual \& Motor Skills, vol. 68, no. 3, pp. 1199-1204, 1989.

[26] C. Rorden and H.-O. Karnath, "Using human brain lesions to infer function: a relic from a past era in the fMRI age?" Nature Reviews Neuroscience, vol. 5, no. 10, pp. 813-819, 2004.

[27] C. Rorden, H.-O. Karnath, and L. Bonilha, "Improving lesionsymptom mapping," Journal of Cognitive Neuroscience, vol. 19, no. 7, pp. 1081-1088, 2007.

[28] C. Rorden, J. Fridriksson, and H.-O. Karnath, "An evaluation of traditional and novel tools for lesion behavior mapping," NeuroImage, vol. 44, no. 4, pp. 1355-1362, 2009.

[29] H.-O. Karnath and J. P. Steinbach, "Do brain tumours allow valid conclusions on the localisation of human brain functions?-objections," Cortex, vol. 47, no. 8, pp. 1004-1006, 2011.

[30] L. S. Radloff, "The CES-D Scale: a self-report depression scale for research in the general population," Applied Psychological Measurement, vol. 1, no. 3, pp. 385-401, 1977.

[31] R. C. Oldfield, "The assessment and analysis of handedness: the Edinburgh inventory," Neuropsychologia, vol. 9, no. 1, pp. 97-113, 1971.

[32] M. F. Folstein, S. E. Folstein, and P. R. McHugh, "MINIMENTAL STATE. A practical method for grading the cognitive state of patients for the clinician," Journal of Psychiatric Research, vol. 12, pp. 189-198, 1975.

[33] H. E. Nelson, "A modified card sorting test sensitive to frontal lobe defects," Cortex, vol. 12, no. 4, pp. 313-324, 1976.

[34] S. Aschenbrenner, O. Tucha, and K. W. Lange, Regensburger Wortflüssigkeits-Test, Hogrefe, Göttingen, Germany, 2000.

[35] K.-H. Schmidt and P. Metzler, Wortschatztest [Vocabulary Test] (WST), Beltz Test, Weinheim, Germany, 2008.

[36] M. Brett, A. P. Leff, C. Rorden, and J. Ashburner, "Spatial normalization of brain images with focal lesions using cost function masking," NeuroImage, vol. 14, no. 2, pp. 486-500, 2001.

[37] E. Brunner and U. Munzel, "The nonparametric Behrens-Fisher problem: asymptotic theory and a small-sample approximation," Biometrical Journal, vol. 42, no. 1, pp. 17-25, 2000.

[38] D. Y. Kimberg, H. B. Coslett, and M. F. Schwartz, "Power in voxel-based lesion-symptom mapping," Journal of Cognitive Neuroscience, vol. 19, no. 7, pp. 1067-1080, 2007.

[39] A. Kiesel, M. Steinhauser, M. Wendt et al., "Control and interference in task switching-a review," Psychological Bulletin, vol. 136, no. 5, pp. 849-874, 2010.

[40] T. Shallice, D. T. Stuss, T. W. Picton, M. P. Alexander, and S. Gillingham, "Mapping task switching in frontal cortex through neuropsychological group studies," Frontiers in Neuroscience, vol. 2, pp. 79-85, 2008.

[41] A. R. Aron, S. Monsell, B. J. Sahakian, and T. W. Robbins, "A componential analysis of task-switching deficits associated with lesions of left and right frontal cortex," Brain, vol. 127, no. 7, pp. 1561-1573, 2004.

[42] T. Shallice, D. T. Stuss, T. W. Picton, M. P. Alexander, and S. Gillingham, "Multiple effects of prefrontal lesions on taskswitching," Frontiers in Human Neuroscience, vol. 1, article 2, 2008.

[43] A. Vallesi, "Organisation of executive functions: hemispheric asymmetries," Journal of Cognitive Psychology, vol. 24, no. 4, pp. 367-386, 2012. 
[44] B. Kopp, N. Rösser, S. Tabeling et al., "Disorganized behavior on Link's cube test is sensitive to right hemispheric frontal lobe damage in stroke patients," Frontiers in Human Neuroscience, vol. 8, article 79, 2014.

[45] J. D. Henry and J. R. Crawford, "A meta-analytic review of verbal fluency performance following focal cortical lesions," Neuropsychology, vol. 18, no. 2, pp. 284-295, 2004.

[46] J. V. Baldo, S. Schwartz, D. Wilkins, and N. F. Dronkers, "Role of frontal versus temporal cortex in verbal fluency as revealed by voxel-based lesion symptom mapping," Journal of the International Neuropsychological Society, vol. 12, no. 6, pp. 896-900, 2006.

[47] J. V. Baldo, A. P. Shimamura, D. C. Delis, J. Kramer, and E. Kaplan, "Verbal and design fluency in patients with frontal lobe lesions," Journal of the International Neuropsychological Society, vol. 7, no. 5, pp. 586-596, 2001.

[48] N. Geschwind, "Disconnexion syndromes in animals and man," Brain, vol. 88, no. 3, p. 585, 1965.

[49] D. T. Stuss and R. T. Knight, Principles of Frontal Lobe Function, Oxford University Press, New York, NY, USA, 2nd edition, 2013.

[50] A. R. Aron, "The neural basis of inhibition in cognitive control," Neuroscientist, vol. 13, no. 3, pp. 214-228, 2007.

[51] R. T. Knight and M. D’Esposito, "Lateral prefrontal syndrome: a disorder of executive control," in Neurological Foundations of Cognitive Neuroscience, M. D’Esposito, Ed., pp. 259-279, MIT Press, Cambridge, Mass, USA, 2003. 


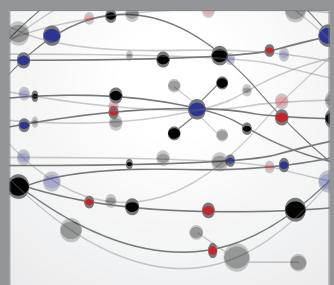

The Scientific World Journal
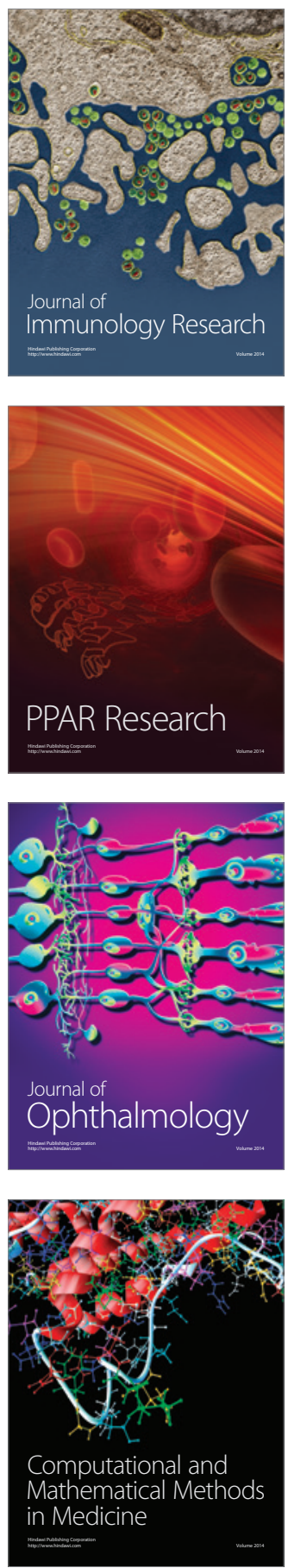

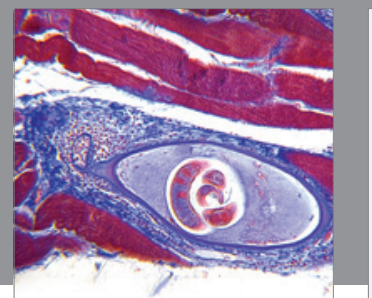

Gastroenterology

Research and Practice
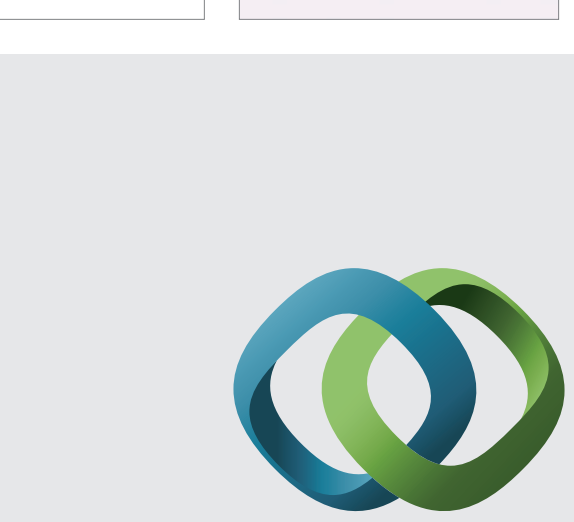

\section{Hindawi}

Submit your manuscripts at

http://www.hindawi.com
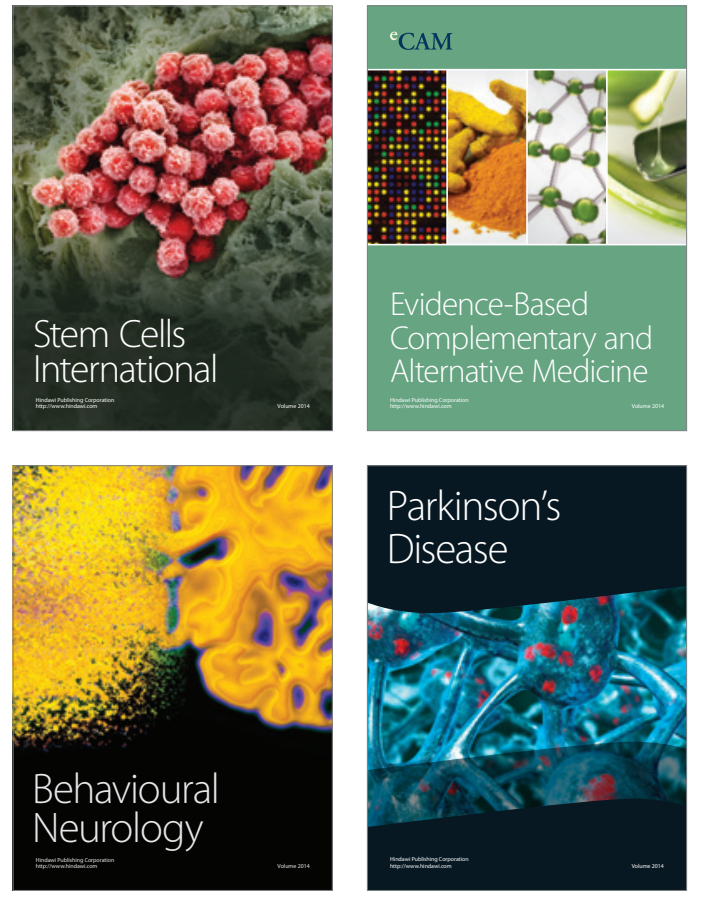
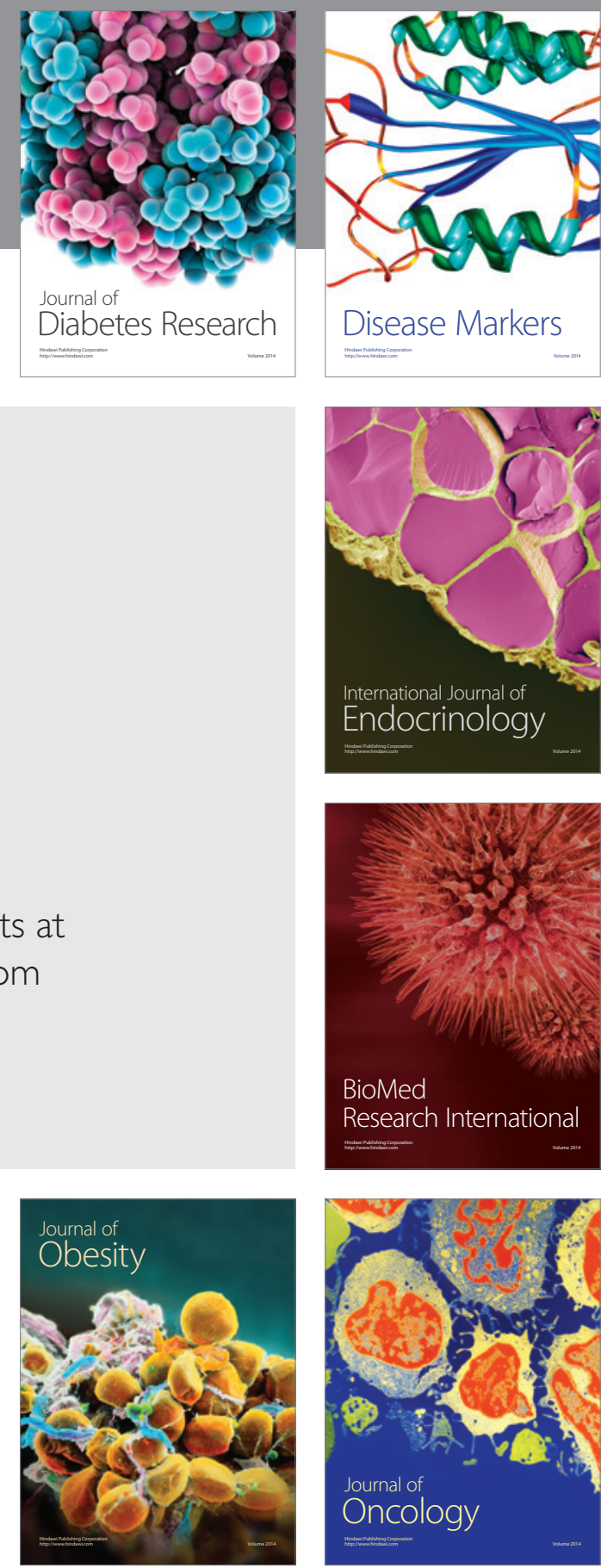

Disease Markers
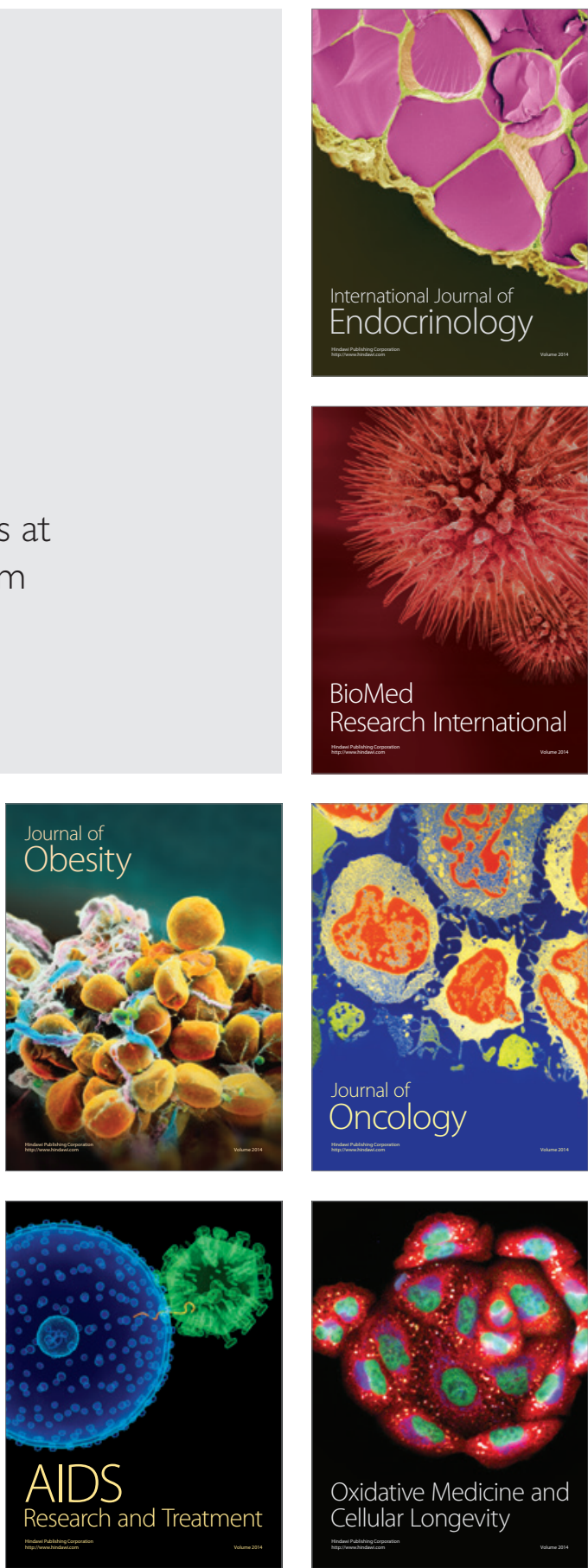